höhere Ebene gelangen sie schliełlich, in dem sie für die arbeitenden Menschen nicht nur Deklaration, sondern Realität werden. In diesem Sinne hat Rosa Luxemburg formuliert:

.,Es gilt, an den durch die bürgerlichen Klassen anderthalb Jahrhunderte lang mißbrauch. ten Woitten die praktische Kritik historischer Handlungen zu üben. Es gilt, die ,Liberté, Egalitê, Fraternite", die 1789 in Frankreich vom Bürgertum proklamiert worden ist, zum erstenmal zur Wahrheit zu machen - durch die Abschaffung der Klassenherrschaft des Bürgertums. " (14)

14 s. Rosa Luxemburg: Nationalversammlung oder Rätegregierung? in: Die Rote Fahne (Berlin) Nr. 32 lom 17. Dezember 1918, hier zit. nach Gesammelte Werke Band 5, Berlin (DDR) 1974 , S. 465

\title{
Tagungsbericht: Internationale Konferenz ,Sozialismus in der gegenwärtigen Welt", Cavtat (Jugoslawien), 27.9. - 2.10.1976
}

Die jugoslawischen marxistischen Zeitschriften „Marksizam u Svetu“ (Marxismus in der Welt) und "Komun ist" hatten eingeladen: neben jugoslawischen Intellektuellen Repräsentanten kommunjstischer, sozialistischer und sozialdemokratischer Parteien aus den entwickelten kapitalisti. schen Ländern (vor allem Westeuropas), aus den sozialistischen Ländern Osteuropas und aus einigen der aus dem antikolonialen Befreiungskampf hervorgegangenen jungen Nationalstaaten. Schlietslich war eine Reihe ,freischwebender" marxistischer Theoretiker und Vertreter theoretischer marxistischer Zeitschriften geladen worden (so auch die Berichterstatter). Das ,politische Gewicht" der Tejlnehmer und ihrer Vorträge war entsprechend unterschiedlich. Die Konferenz muf auf dem Hintergrund des Wandlungsprozesses gesehen werden, den die kommunistische Weltbewegung und die sozialistischen Parteien sowie das Verhältnis der beiden Fraktionen der Arbeiterbewegung in den letzten Jahren durchgemacht haben bzw. in einigen Ländern durchzumachen im Begriff sind. Insofern schien die Konferenz von Cavtat fast wie eine unverbindliche (es gab kein Kommunique), weniger prominente, dafür auf die nichtkommunistischen Fraktionen der in ternationalen Arbeiterbewegung ausgeweitete Variante der Berliner Konferenz der europäischen Kommunisten vom Juni 1976.

Politisch bezweckten die jugoslawischen Veranstalter offenbar u.a. folgendes: Demonstration des Willens, den eigenen Weg zum Sozialismus weiterzugehen, wobei man sich der zunehmenden Unterstützung der westeuropäischen kommunistischen Massenparteien und der jungen Nationalstaaten versichern will; Propagierung der Einsicht in die strategische Notwendigkeit, die historische Spaltung der Arbeiterbewegung zu entschärfen; Gelegenheit zur Selbstdarstellung einer weltweiten Mannigfaltigkeit der Positionen, die einseitige Bind ungen an ein einziges Zentrum kaum mehr zulassen. Innerjugoslawisch konnte man sich von der Konferenz Impulse gegen bürgerliche Tendenzen in Wissenschaft und Bildung versprechen. Schließlich mag es nicht zufällig sein, daß die Konferenz nach der Einstellung der "Sommerschule“" von Korcula als neues jährlich stattfindendes Forum gegründet wurde. (Die Konferenzen von Korcula wurden viele Jahre lang von der sog. Praxis-Gruppe veranstaltet, und dann im Verlauf der Auseinandersetzungen mit dieser Gruppe eingestellt.)

Die Konferenz gliederte sich in drei Themenschwerpunkte:

1. Die Veränderungen des gegenwärtigen Kapitalismus und die Probleme sozialistischer Strategie.

2. Probleme der antikolonialen und antiimperialistischen Revolution.

3. Probleme des Sozialismus und der Rolle der Arbeiterklasse.

Im Mittelpunkt der Strategiediskussion standen Fragen der politischen Form der Entmach- 
tung der Bourgeoisie. Dabei wurde die historische Hoffnung der Arbeiterklasse ausgedrückt, als Klasse, die die "Zukunft in ihren Händen trägt" (Kommunistisches Manifest), die fortschrittlichen Errungenschaften der bürgerlichen Gesellschaft dort bewahren und weiterentwickeln zu können, wo die Bourgeoisie selbst dazu nicht mehr in der Lage ist. In der zunehmenden Unfähigkeit auch der sozialdemokratisch integrationistischen Parteien, eine glaubwürdige Perspektive demokratischer Gesellschaftsentwicklung zu repräsentieren, und im Prozess der abnehmenden In tegrationsfähigkeit der Großbourgeoisie gegenüber den Mittelschichten und Teilen der noch vorhandenen Kleinbourgeoisie wurde der Ansatz sozialistisch-demokratischer Strategiebildung gesehen, die zunehmend auch die Gegensätze innerhalb der Arbeiterbewegung abzubauen in der Lage ist. Auf den ersten Blick machte die Konferenz in diesem Abschnitt, in dem naturgemäß vor allem die Vertreter der kommunistischen und sozialistischen Parteien Westeuropas den Ton angaben, den Eindruck, als gäbe es hier kaum Kontroversen oder ungelöste Probleme. Sozialisten und Kommun isten kamen dar in überein, Pluralismus und Demokratie als Elemente des revolutionären Weges in den kapitalistischen Ländern einzuschätzen: Beibehaltung und Weiterentwicklung der existierenden Formen der bürgerlichen Demokratie, Sicherung der bürgerlichen Freiheiten, des Koalitionsrechts, des Parteienpluralismus und der Abwählbarkeit der Regierung. Darüber hinaus schien es zeitweilig, als würden sich die Vertreter der einzelnen Parteien jeweils auf Fragen konzentrieren, die bei ihnen traditionell eher vernachlässigt worden waren: die Kommunisten auf die Frage der politischen Demokratie und ihrer Vermittlung mit Formen der Basisdemokratie in der Produktion, die Sozialisten auf Fragen der Entmachtung der Bourgeoisie. Von beiden Seiten wurde hervorgehoben, daß Prozesse weitergehender Demokratisierung in den sozialistischen Ländern eine wesentliche Ǔnterstützung der sozialistischen Bewegungen vor allem Westeuropas darstellen könnten. Gegen den Zielbegriff der ,Einheit" und für den des ,Bündnisses" sprach sich J. P. Cotten von der KP Frankreichs aus mit der Begründung, dał das Bündnis Gegensätze in sich aufnehmen und er tragen kann.

Der Exekutivsekretär der chilenischen Unidad Popular, Chlodomiro Almeyida, mahnte ebenso wie andere Sprecher der sozialistischen Bewegungen Lateinmaerikas und ein sowjetischer Delegier ter, Fragen der konterrevolutionären Gewalt und der Verteidigungsmacht für einen demotischen und sozialistischen Staat nicht zu vernachlässigen. Eine kurze Kontroverse gab es zwischen den Lateinamerikanern und Lelio Basso, der beschworen wurde, den Faschismusbegriff nicht im Blick auf den italienischen und den deutschen Faschismus zu eng zu definieren und vor allem die Massenbewegung nicht zum starren Kriterium zu machen. Der Faschismusbegriff müsse z. B. auf die Verhältnisse in Chile anwendbar sein, schon seiner internationalen Verständlichkeit wegen. „Desto in ternationaler ist der Kampf dagegen “, sagte Poblo Gonzalez Casanova aus Mexiko. Faschismus komme heute auch ohne Massenbewegung aus. Die gewaltlose Verteidigung demokratischer Freiheiten allein löse nicht das Problem, wie faschistischen Putschen entgegenzutreten werden kann.

Wurden die repräsentativen Positionen wesentlich anhörend, ohne Kontroverse rezipiert - wenn man von jugoslawisch-sowjetischen Wortwechseln über die Bedeutung von Selbstverwaltung bzw. Staatseigentum absieht -, so gab es scharfe Wortgefechte um Thesen gerade solcher Intellektueller, die einen individuellen wissenschaftlichen Ansatz repräsentierten, ohne diesen mit einer politischen Bedeutung innerhalb der sozialistischen Bewegung ihrer Länder verbinden zla können. Scharfe Auseinandersetzungen fanden vor allem um die wissenschaftlichen Grundlagen und strategischen Konsequenzen der Vorstellungen Arghiri Emanuels statt, der die Arbeiterklasse der entwickelten kapitalistischen Länder als keinen Mehrwert produzierend, sondern yom neokolonial, (durch ungleichen Tausch im Rahmen imperialistischer Machtverhältnisse) angeeigneten Mehrwert der ,unterentwickelten " Länder zehrend und daher absolut unfähig zur sozialistischen Transformation ihrer Länder bezeichnete. Diese Position wurde sowohl von den Westeuropäern als auch von den Organisationssprechern der jungen Nationalstaaten zurückgewiesen. Dennoch ist die Heftigkeit, mit der diese Zurcükweisung erfolgte, ein Anzeichen rür unerledigte Probleme und Widersprüche der sozialistischen Weltbewegung, die noch kaum voll begriffen sind. Wie ist es in den entwickelten kapitalistischen Ländern so lange zu einem Stillstand der sozialistischen Bewegung gekommen? Die Lücken in - oder gar das Fehlen - einer internationalen Diskussion über die Ursachen sozialdemokratischer Integration der Arbeiterbewegung wurde hier 
deutlich. Ferner wurde der gedankenlose Europäozentrismus mit Recht attackiert. In überspitzter Form sprach Anouar Abdel Malek von der Marginalität Europas. Die sog. Dritte Welt sei in Wirklichkeit die Erste. Auch ein mangelndes Verständ nis heute möglicher sozialistischer Entwicklungen ohne das kapitalistische Zwischenstadium mag hier berührt worden sein.

Weitere (möglicherweise unvermeidliche) Schwächen der Konferenz seien wenigstens angedeu tet: Zu den Problemen, die in den Beiträgen zur Wiedergewinnung einer historischen Handlungsfähigkeit der Arbeiterbewegung in Westeuropa ausgeklammert wurden, gehörte vor allem die Frage der ökonomischen Strategie. Undiskutiert blieb z. B, wie sich in der Krise die auf Verteidigung der Lebensbedingungen zielende Gewerkschaftspolitik zu der auf sozialistische Bündnistrategie angelegten Politik der Parteien verhalten soll. Schlieflich bilden solche Probleme gegenwärtig die Grundlage wesentlicher Konflikte in der italienischen KP. - Von vielen der Beteiligten - auch von den meisten jugoslawischen Teilnehmern - wurde an der Verschiebung des Machtschwerpunkts in der Bundesrepublik nach rechts geflissentlich vorbeigesehen. - Viele der in der Übersetzung nur in Bruchstücken verständlichen, zu langen und zu schnell gesprochenen jugsolawischen Beiträge klammerten die beträchtlichen ökonomischen, sozialen und politischen Probleme des eigenen Landes aus zugunsten mehr ,ideologischer" Rechtfertigung des eignen Modells. Dabei wurde viel Marx zitiert - was der spanische Kommunist Comin monierte - ohne die unterschiedliche Bedeutung des Begriffs "Selbstverwaltung der Gesellschaft", die bei Marx und Engels den Staat absterben macht, und "Selbstverwaltung" im Sinne der politischen und ökonomischen Strukturen Jugoslawiens zu reflektieren.

Diese Kritik mindert in keiner Weise die Bedeutung und den großen Nutzen der Konferenz. Es zeigte sich,daß der Wandlungsprozess der internationalen Arbeiterbewegung, die Entschärfung der Gegensätze einhergehen mit der Wiedergewinnung einer weniger beschränkten und desto mehr konsistenten wissenschaftlichen Diskussionsfähigkeit. Man spürte förmlich das lange aufgestaute Bedürfnis nach dieser Art von Kommunikation. Es herrschte in keiner Weise die sprichwörtiche Kongresslangeweile. Nicht zuletzt für die Teilnehmer aus der Bundesrepublik, einem Land mit sich stürmisch entwickelnder Rolle im imperialistischen Weltsy stem und desto unentwickelter sozialer Bewegung (von Oertzen hatte es für die SPD bezeichnenderweise abgelehnt, teilzunehmen), war die Tagung außerordentlich nützlich für die fällige Entprovinzialisierung des eigenen Horizonts.

(E. Altvater, W. F. Haug, Chr. Neusüß)

Aufgrund eines technischen Versehens sind eine Reihe Fahnenkorrekturen bei dem Aufsatz von P. Brandt in Nr. 26 nicht berücksichtigt worden. Wir berichtigen im folgenden die dadurch entstandenen Fehler außer Druckfehlern, die eindeutig als solche erkennbar sind:

$S .40$, vor Anm. 10: statt ,etwa die Hälfte" ": ,die relative Mehrheit".

Anm. 24: Flechtheim, S. $171 \mathrm{ff}$; Rosenberg, S. $125 \mathrm{ff}$.

Anm. 28: Für die SPD ..., S. 119 ff., und S. Neumann . ., S. 32 ff (hier S. 33).

Anm. 40: Rundschau Nr. 14/1934.

Anm. 48: Past and Present 33 (1966).

Anm. 78: gehört an das Ende des auf Anm. 77 folgenden Satzes (S. 70). Anstelle von Anm. 78 gehört

Anm. 79: Für Deutschland vor allem K. H. Roth (Anm. 58). Sein Buch wurde ausführlich in der Zeitschrift „Politikon“ diskutiert. 